\title{
Response of Glucocorticoid Receptor Alpha and Histone Deacetylase 2 to Glucocorticoid Treatment Predicts the Prognosis of Sudden Sensorineural Hearing Loss
}

\author{
Xiuling Zhang ${ }^{1,2}$ (B) $\cdot$ Jinxiang Chen ${ }^{2} \cdot$ Ziwen Gao $^{1} \cdot$ Hui Qi $^{3} \cdot$ Yanhong Dai ${ }^{1,3} \cdot$ Wandong She ${ }^{1,3}$ (]) \\ ${ }^{I}$ Department of Otolaryngology-Head and Neck Surgery, Nanjing Drum Tower Hospital Clinical College of Nanjing Medical University, Nanjing \\ City; ${ }^{2}$ Department of Otolaryngology-Head and Neck Surgery, The Affiliated Jiangyin Hospital of Southeast University Medical School, Jiangyin \\ City; ${ }^{3}$ Department of Otolaryngology-Head and Neck Surgery, Nanjing Drum Tower Hospital, The Affiliated Hospital of Nanjing University
}

Medical School, Nanjing City, China

Objectives. To investigate glucocorticoid receptor (GR) and histone deacetylase 2 (HDAC2) gene expression and protein levels in peripheral blood mononuclear cells (PBMCs) of patients with severe or profound sudden sensorineural hearing loss (SSNHL) and to explore the roles of GRs and HDAC2 in glucocorticoid (GC) insensitivity.

Methods. Fifty-five severe or profound SSNHL patients were enrolled in the study. According to hearing improvement after GC treatment, patients were assigned into two groups: GC-sensitive and GC-resistant. A normal reference group included 20 healthy volunteers without hearing loss. Quantitative real-time polymerase chain reaction and Western blot analyses were used to detect the relative expression of GR $\alpha$, GR $\beta$, and HDAC2 in PBMCs at the mRNA and protein levels.

Results. The protein levels of GRs and HDAC2 in PBMCs of SSNHL patients were lower than the normal reference values before GC treatment. Compared with the GC-resistant group, both the mRNA and protein levels of GR $\alpha$ and HDAC2 were significantly increased in the GC-sensitive group after GC treatment.

Conclusion. A lack of GR $\alpha$ and HDAC2 induction following steroid treatment in GC-resistant SSNHL patients may play a fundamental mechanistic role in GC insensitivity. Response of GR $\alpha$ and HDAC2 to steroid treatment may, thus, predict the prognosis of hearing improvement in SSNHL patients.

Keywords. Sudden Sensorineural Loss; Glucocorticoid Receptor; Deacetylase 2; Prognosis

\section{INTRODUCTION}

Glucocorticoids (GCs) are currently the major drug in the treatment of certain types of inner ear disorders, such as sudden sen-

\footnotetext{
- Received September 9,2018

Revised December 19, 2018

Accepted January 3, 2019

- Corresponding author: Wandong She

Department of Otolaryngology-Head and Neck Surgery, Nanjing Drum

Tower Hospital Clinical College of Nanjing Medical University,

321 Zhongshan Road, Nanjing City, Jiangsu Province 210008, China

Tel: +86-25-6818-2139, Fax: +86-25-8331-7016

E-mail: shewandong@163.com
}

sorineural hearing loss (SSNHL), noise-induced hearing loss, Meniere's disease and cisplatin-induced ototoxicity [1,2]. They are particularly recommended as the first line treatment for SSNHL in clinical practice guidelines in many countries [3,4]. It has been reported that GC can decrease inflammation, increase the expression of antioxidant enzymes, and reduce apoptosis induced by ototoxicity and SSNHL in the cochlea [2,5].

GCs bind to glucocorticoid receptors (GRs) to provide antiinflammatory and anti-apoptotic functions in the target tissues. GRs belong to the nuclear hormone receptor superfamily and contain three major functional domains: a N-terminal transactivation domain, a central DNA-binding domain, and a C-termi-

Copyright (C) 2019 by Korean Society of Otorhinolaryngology-Head and Neck Surgery.

This is an open-access article distributed under the terms of the Creative Commons Attribution Non-Commercial License (http://creativecommons.org/licenses/by-nc/4.0)

which permits unrestricted non-commercial use, distribution, and reproduction in any medium, provided the original work is properly cited. 
nal ligand-binding domain [6]. The GRs have two major isoforms, GR $\alpha$ and GR $\beta$, which are different in the final coding exon. GRa is thought to be the main mediator of GC action while GR $\beta$ is considered as an antagonist of GR $\alpha$ [7]. GR $\alpha$ and GR $\beta$ are ligand-dependent transcription factors and expressed in most cell types, however, GR $\beta$ is generally expressed at low levels compared to GR $\alpha$ [7]. In the human inner ear, the distribution of GRs is widespread, and the highest concentration is observed in the spiral ligament [8].

Although most SSNHL patients respond well to GC treatment, about $20 \%$ of SSNHL patients show little or no response to GC treatment, i.e., GC-resistance [9]. Mechanisms of GC-resistance are still unknown. Recent studies suggest that changes in cellular micro-environment, induction of cytokines as a result of chronic inflammation, hypoxia, oxidative stress, allergen exposure and serum-derived factors may contribute to the development of GC resistance [10]. Beside these mechanisms, an accumulating body of evidence indicates that posttranslational modification of GRs and reduced histone deacetylase 2 (HDAC2) activity are also involved in steroid resistance [11]. Some studies indicate that upregulation of GR $\alpha$ and downregulation of GR $\beta$ can improve steroid sensitivity $[12,13]$. This model is supported by observations that histone deacetylases are recruited by GRs for transrepression [14]. A recent study also suggests that GRa increases HDAC2 promoter activity, while GR $\beta$ abolishes this effect in a dose-dependent manner [11]. Our previous study indicated that reduced HDAC2 protein level may be one of the mechanisms of GC insensitivity in patients with refractory SSNHL [15]. In this study, we further explored the roles of HDAC2 and two isoforms of GR in GC sensitivity in patients with severe and profound SSNHL.

\section{MATERIALS AND METHODS}

\section{Subjects}

The study was conducted at The Affiliated Jiangyin Hospital of Southeast University Medical School from January 2015 to October 2017. The study protocols were approved by the hospital Ethics Committee (No. ChiCTR-BOC-16008999).

Fifty-five SSNHL patients with severe or profound hearing loss (pure tone audiometry [PTA] at $0.5-4 \mathrm{kHz} \geq 61 \mathrm{~dB}$ within

\section{H I G G H L I I G H T T S}

- Glucocorticoid receptor (GR) and histone deacetylase 2 (HDAC2) were assayed in sudden sensorineural hearing loss (SSNHL) patients.

- Increased levels of GR and HDAC2 were observed in glucocorticoid-sensitive patients.

- The response of GR $\alpha$ and HDAC2 to steroid predicts hearing gain in SSNHL patients.
72 hours of onset according to American Academy of Otolaryngology-Head and Neck Surgery guidelines) [3] were included in the study. Exclusion criteria included middle or external ear diseases or middle ear surgery history, retrocochlear diseases, malignancy or autoimmune diseases or head trauma history, or a history of asthma or chronic obstructive pulmonary disease, or a family history of hearing loss. Subjects pretreated with GCs or other medicines before this study were also excluded. Informed consent documentation was obtained from all patients before treatment.

All patients received a conventional therapy, including systemic GC (methylprednisolone sodium succinate, $80 \mathrm{mg}$ /day for 4 days, then $40 \mathrm{mg} /$ day for 3 days, and $20 \mathrm{mg} /$ day for another 3 days; Pfizer Manufacturing Belgium NV, Puurs, Belgium), antioxidant $(105 \mathrm{mg} /$ day for 10 days, Ginkgo biloba extract injection; Dr. Willmar Schwabe GmbH \& Co. KG, Essen, Germany), and mecobalamin injection $(500 \mu \mathrm{g} /$ day for 10 days; Yangtze River Pharmaceutical Group, Nanjing Hailing Pharmaceutical, Nanjing, China). PTA was measured before and 24 hours after the 10-day conventional treatment, as well as during a 3-month follow-up period. According to hearing improvement at 0.25 $8 \mathrm{kHz}$, patients were assigned into two groups: a GC-sensitive group (hearing improvement $\geq 15 \mathrm{~dB}$, GCS group) and a GCresistant group (hearing improvement $<15 \mathrm{~dB}$, GCR group) according to the guideline published by Editorial Board of Chinese Journal of Otorhinolaryngology-Head \& Neck Surgery and Chinese Medical Association [16]. Twenty healthy volunteers without hearing loss were also enrolled in the study as a normal reference group (NR group) to obtain the normal reference levels of GRs and HDAC2 in peripheral blood mononuclear cells (PBMCs).

\section{Preparation of PBMCs}

Peripheral blood was collected before and 24 hours after the 10day conventional GC treatment. PBMCs were isolated by the Ficoll-Paque Plus density-gradient method (GE Healthcare Biosciences, Uppsala, Sweden) and stored at $-80^{\circ} \mathrm{C}$ until RNA and protein extractions (detailed below).

\section{Quantitative real-time polymerase chain reaction}

The mRNA levels of GR $\alpha$, GR $\beta$ and HDAC2 were measured by quantitative real-time polymerase chain reaction (qPCR). Total RNA was isolated from PBMCs by adding $1 \mathrm{~mL}$ Trizol (Invitrogen, Waltham, MA, USA) and then $200 \mu \mathrm{L}$ chloroform. RNA was used to synthesize single-stranded cDNA by the Prime Script RT-PCR Kit (Takara, Kusatsu, Japan) according to the manufacturer's protocol. qPCR was performed with SYBR Green PCR Master Mix Reagent (Takara). The Applied Biosystems 7500 real-time PCR system (Applied Biosystems, Foster City, CA, USA) was used to perform real-time PCR. The primers were designed by Primer Bank (Table 1). The thermal cycle conditions were consisted of one 30-second cycle of predenaturation at $95^{\circ} \mathrm{C}$ followed by 40 cycles of 5 -second denaturation at 
Table 1. Primers used for qPCR

\begin{tabular}{ll}
\hline Primer & \multicolumn{1}{c}{ Sequence } \\
\hline hGR $\alpha$ upstream primer & 5'-GAAGGAAACTCCAGCCAGAAC-3' \\
hGR $\alpha$ downstream primer & 5'-CTGATTGGTGATGATTCAGCTA-3' \\
hGR $\beta$ upstream primer & 5'-CCTAAGGACGGTCTGAAGAGC-3' \\
hGR $\beta$ downstream primer & 5'-CCACGTATCCTAAAAGGCAC-3' \\
HDAC2 upstream primer & 5'-ATGGCGTACAGTCAAGGAGG-3' \\
HDAC2 downstream primer & 5'-TGCGGATTCTATGAGGCTTCA-3' \\
GAPDH upstream primer & 5'-CTGGGCTACACTGAGCACC-3' \\
GAPDH downstream primer & 5'-AAGTGGTCGTTGAGGGCAATG-3' \\
\hline
\end{tabular}

qPCR, quantitative real-time polymerase chain reaction; hGR, human glucocorticoid receptor; HDAC2, histone deacetylase 2; GAPDH, glyceraldehydes-3-phosphate dehydrogenase.

$95^{\circ} \mathrm{C}$ and 30 -second annealing/elongation at $60^{\circ} \mathrm{C}$. Melt curve analysis was performed to ensure the amplification of a single product. Target mRNA expression levels were calculated by the 2-( $\Delta \Delta \mathrm{Ct})$ method [17].

\section{Western blot analysis}

The protein levels of GR $\alpha$, GR $\beta$, and HDAC 2 were measured by Western blot. To extract total protein, cells were homogenized in ice-cold radio immunoprecipitation assay Lysis Buffer (Beyotime, Nanjing, China) containing $1 \mathrm{mM}$ phenylmethylsulfonyl fluoride. Debris was removed by centrifuging at $13,000 \times g$ for 5 minutes at $4^{\circ} \mathrm{C}$. Protein concentrations were determined by using the BCA protein assay kit (Beyotime). Thirty-five micrograms of total protein from each sample were resolved by SDS-PAGE and then transferred to a nitrocellulose membrane. Polyclonal rabbit antiGR $\alpha$ antibody (1:500, ab3580; Abcam, Cambridge, UK), polyclonal rabbit anti-GR $\beta$ antibody (1:500, ab3581; Abcam), or monoclonal rabbit anti-HDAC2 antibody (1:1,000, ab32117; Abcam) were used to detect immunoreactive bands of GR $\alpha$, GR $\beta$ and HDAC2, respectively. Goat anti-rabbit horseradish peroxidase-conjugated antibody (1:5,000, ZB-2301; Zsgb-BIO, Beijing, China) or goat anti-mouse horseradish peroxidase-conjugated antibody $(1: 5,000$, ZB-2305; ZSGB-Bio) were used as secondary bodies. Bands of $\mathrm{GR} \alpha, \mathrm{GR} \beta$, and HDAC2 protein were located at 86, 83, and 55 $\mathrm{kD}$ approximately. Immunoreactivity of $\beta$-actin (monoclonal mouse anti- $\beta$-actin, 1:2,000, TA-09; Zsgb-BIO) served as a loading control. Developed X-ray films were scanned, and densitometries of the immunolabeled bands were quantified by using Image J software ver. 1.34 (http://rsb.info.nih.gov/ij/).

\section{Statistical analysis}

All statistical analyses were performed by using SPSS ver. 17.0 (SPSS Inc., Chicago, IL, USA). The qPCR and immunoblot data were presented as the mean \pm standard deviation. Paired samples $t$-test was used to analyze PTA, mRNA expression and protein levels of GR $\alpha, \mathrm{GR} \beta$, and HDAC2 before and after GC treatment. Independent sample $t$-test, one-way analysis of variance test and exact probability in fourfold table were used to determine statis-
Table 2. Clinical characteristics of patients with SSNHL $(n=55)$

\begin{tabular}{|c|c|c|c|c|}
\hline Variable & GCS group & GCR group & $\begin{array}{l}\text { Hearing improvement } \\
\text { rate (\%) }\end{array}$ & $P$-value \\
\hline Sex & & & & 0.50 \\
\hline Male & 17 & 10 & 63.0 & \\
\hline Female & 20 & 8 & 71.4 & \\
\hline Side of lesion & & & & 0.37 \\
\hline Left & 20 & 12 & 62.5 & \\
\hline Right & 17 & 6 & 73.9 & \\
\hline Vertigo & & & & $<0.001$ \\
\hline Yes & 5 & 13 & 27.3 & \\
\hline No & 32 & 5 & 86.5 & \\
\hline Hearing loss & & & & 0.09 \\
\hline Severe ${ }^{b)}$ & 9 & 1 & 90.0 & \\
\hline Profound ${ }^{c)}$ & 28 & 17 & 62.2 & \\
\hline
\end{tabular}

SSNHL, sudden sensorineural hearing loss; GCS, glucocorticoid-sensitive; GCR, glucocorticoid-resistant; PTA, pure tone audiometry.

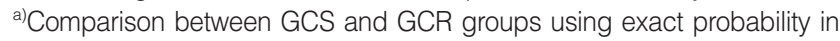

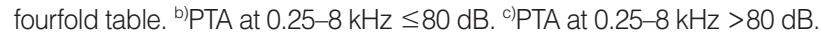

tical difference between two groups. $P$-values less than 0.05 were considered statistically significant.

\section{RESULTS}

\section{Significant hearing recovery in the GCS group}

All SSNHL patients in this study were first diagnosed.Ten SSNHL patients had severe hearing loss (PTA, $61-80 \mathrm{~dB}$ at $0.25-4 \mathrm{kHz}$ ), and 45 patients (approximately $81.82 \%$ ) had profound hearing loss (PTA $>80 \mathrm{~dB}$ at $0.25-4 \mathrm{kHz}$ ) before treatment. According to the recovery of PTA at 3-month follow-up, 37 patients were assigned into the GCS group (hearing improvement $>15 \mathrm{~dB}$ ), and 18 patients into the GCR group (hearing improvement $>15 \mathrm{~dB}$ ). The patients' sex, the side of affected ears and the shape of hearing graph had no significant impact on hearing recovery (all $P>$ $0.05)$ except vertigo $(P<0.001)$ (Table 2$)$, indicating that vertigo is a poor prognostic factor for hearing outcome in SSNHL [18]. No significant differences were observed in the number of patients with profound hearing loss in the two groups $(94.4 \%$ in the GCR group; $75.7 \%$ in the GCS group; $P=0.09$ ) (Table 3). Significant hearing improvement was only observed in the GCS group (PTA, $48.29 \pm 15.30 \mathrm{~dB} ; P<0.001$ ), not in the GCR group (PTA, 87.72 $\pm 11.28 \mathrm{~dB} ; P=0.07)$ after treatment.

\section{Increased mRNA expression of GR $\alpha$ and HDAC2 in the GCS group}

Qualitative evaluations of qPCR amplifications of GR $\alpha$, GR $\beta$, and HDAC2 mRNA in PBMCs revealed single melting curve peaks for each amplicon and single electrophoretic bands for each reaction, corresponding to the predicted size of each product. GR $\alpha$, and HDAC2 mRNAs were detected in all samples. However, GR $\beta$ mRNA was only detected in 87 of the 130 sam- 
Table 3. Hearing loss severity in GCS and GCR groups

\begin{tabular}{lccccc}
\hline & \multicolumn{2}{c}{ GCS group } & & \multicolumn{2}{c}{ GCR group } \\
\cline { 2 - 3 } \cline { 5 - 6 } Initial hearing loss severity & No. of patients $(\%)$ & $\begin{array}{c}\text { PTA gain after treatment } \\
(\mathrm{dB}, 0.25-8 \mathrm{kHz})\end{array}$ & & No. of patients $(\%)$ & $\begin{array}{c}\text { PTA gain after treatment } \\
(\mathrm{dB}, 0.25-8 \mathrm{kHz})\end{array}$ \\
\hline Severe $\mathrm{HL}(61-80 \mathrm{~dB})$ & $9(24.3)$ & $27.63 \pm 10.01$ & $1(5.6)$ & 14.16 \\
Profound $\mathrm{HL}(>80 \mathrm{~dB})$ & $28(75.7)$ & $39.70 \pm 15.28$ & & $17(94.4)$ & $2.11 \pm 5.60$ \\
\hline
\end{tabular}

Values are presented as mean \pm standard deviation.

GCS, glucocorticoid-sensitive; GCR, glucocorticoid-resistant; PTA, pure tone average.
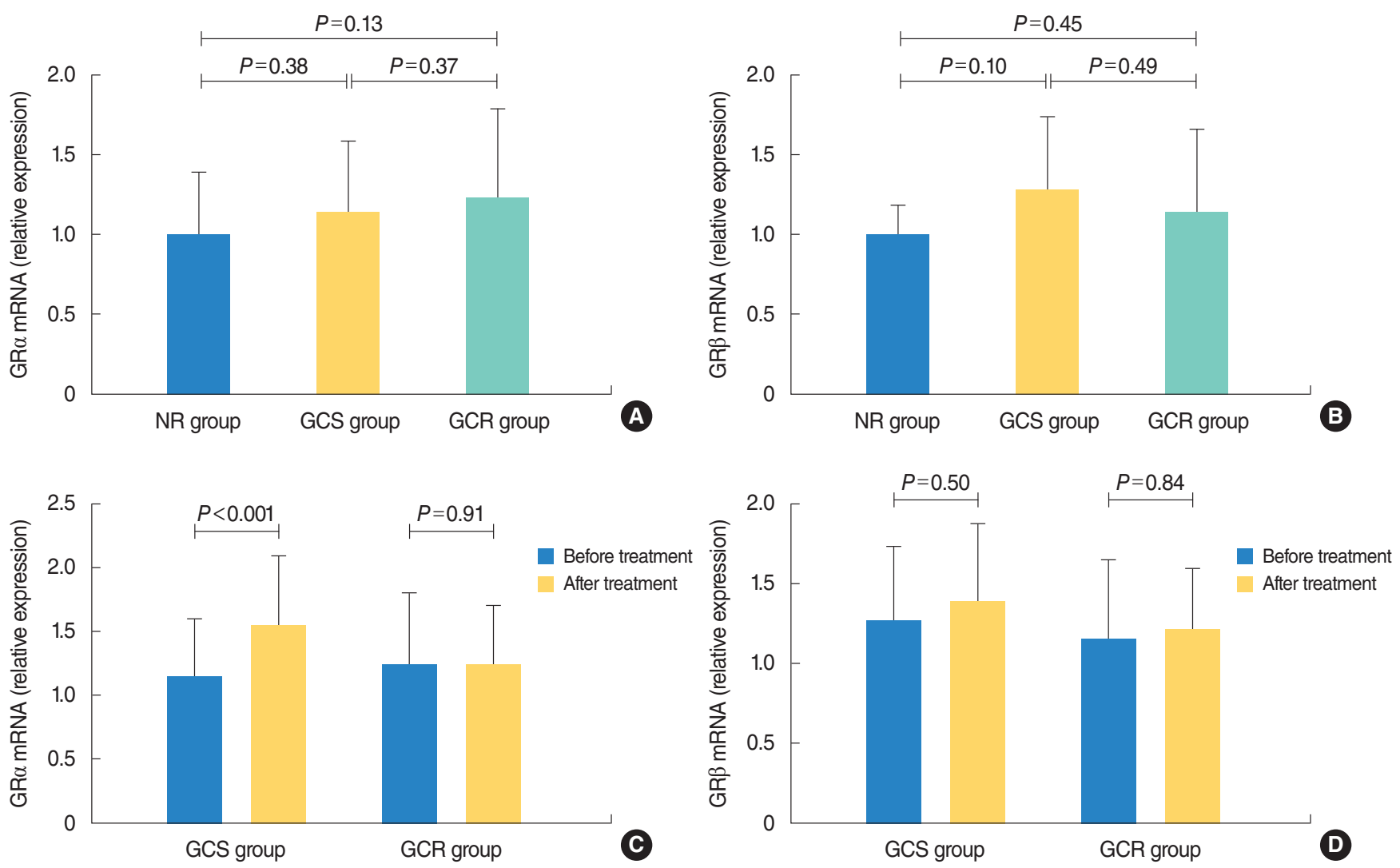

Fig. 1. Glucocorticoid receptor a $(\mathrm{GRa})(\mathrm{A})$ and $\mathrm{GRB}$ mRNA $(\mathrm{B})$ relative expression in the normal reference (NR) group and sudden sensorineural hearing loss patients before treatment. There were no significant differences between groups (all $P>0.05$ ). Comparison of $\mathrm{GR} \alpha(\mathrm{C})$ and GR $\beta$ mRNA (D) relative expression in the glucocorticoid-sensitive (GCS) group and the glucocorticoid-resistant (GCR) group before and after glucocorticoid (GC) treatment. After GC treatment, GRa mRNA expression significantly increased in the GCS group $(P<0.001)$, while no significant change was observed in the GCR group ( $P=0.91)$. After $\mathrm{GC}$ treatment, slightly increased GR $\mathrm{mRNA}$ expression was observed in the GCS and the GCR groups, however, there were no significant differences between groups (all $P>0.05$ ). (A, C) Comparison between groups using one-way analysis of variance test. (C, D) Comparison before and after treatment using paired-sample test.

ples (pretreatment: 31 samples in GCS, 12 samples in GCR groups, 12 samples in NR group; posttreatment: 21 samples in GCS and 11 samples in GCR groups), probably due to a very low-level of GR $\beta$ mRNA in these samples. Eventually, both preand posttreatment GR $\beta$ expression was detected in 16 GCS subjects and $8 \mathrm{GCR}$ subjects and the results were used for a pair comparison. GR $\beta$ mRNA was detected in 12 NR subjects.

Quantitative PCR evaluations revealed that, before GC treatment, all SSNHL patients had equivalent levels of GR $\alpha$, GR $\beta$, and HDAC2 mRNA expressions as the NR group (all $P>0.05$ )
(Figs. 1A, B, and 2A).

After GC treatment, GR $\alpha$ and HDAC2 mRNA expression levels were all increased in the GCS group (GR $\alpha, P<0.001$; HDAC2, $P<0.001$ ) (Figs. 1C, 2B). However, such increase was not observed in the GCR group (GR $\alpha, P=0.91 ; \mathrm{HDAC} 2, P=0.52)$ (Figs. $1 \mathrm{C}, 2 \mathrm{~B})$. There was a slightly increased GR $\beta$ mRNA expression in both the GCS and GCR groups after treatment compared to the levels before treatment, but no significant differences were observed (all $P>0.05$ ) (Fig. 1D). 

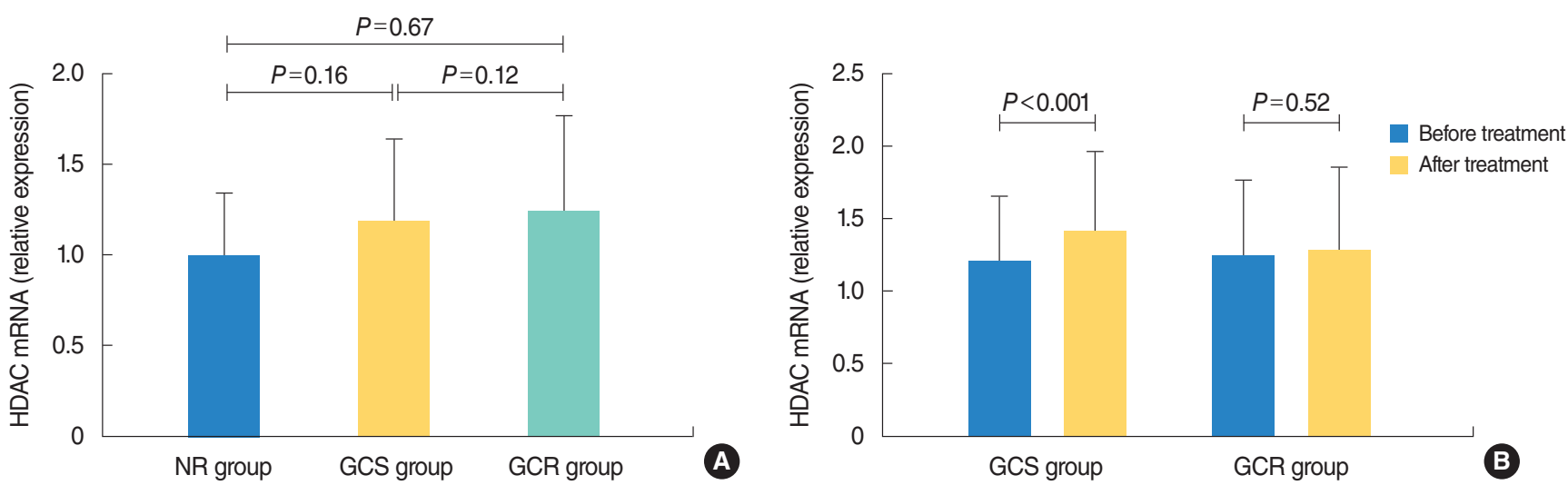

Fig. 2. (A) Histone deacetylase 2 (HDAC2) mRNA relative expression in normal reference (NR) group and sudden sensorineural hearing loss (SSNHL) patients before glucocorticoid (GC) treatment. There were no significant differences between groups. (B) Comparison of HDAC2 mRNA relative expression in glucocorticoid-sensitive (GCS) group and glucocorticoid-resistant (GCR) group before and after GC treatment. After GC treatment, the HDAC2 mRNA expression significantly increased in the GCS group $(P<0.001)$ while no significant change was observed in the GCR group $(P=0.52)$. (A) Comparison between groups using one-way analysis of variance test. (B) Comparison before and after treatment using paired-sample test.
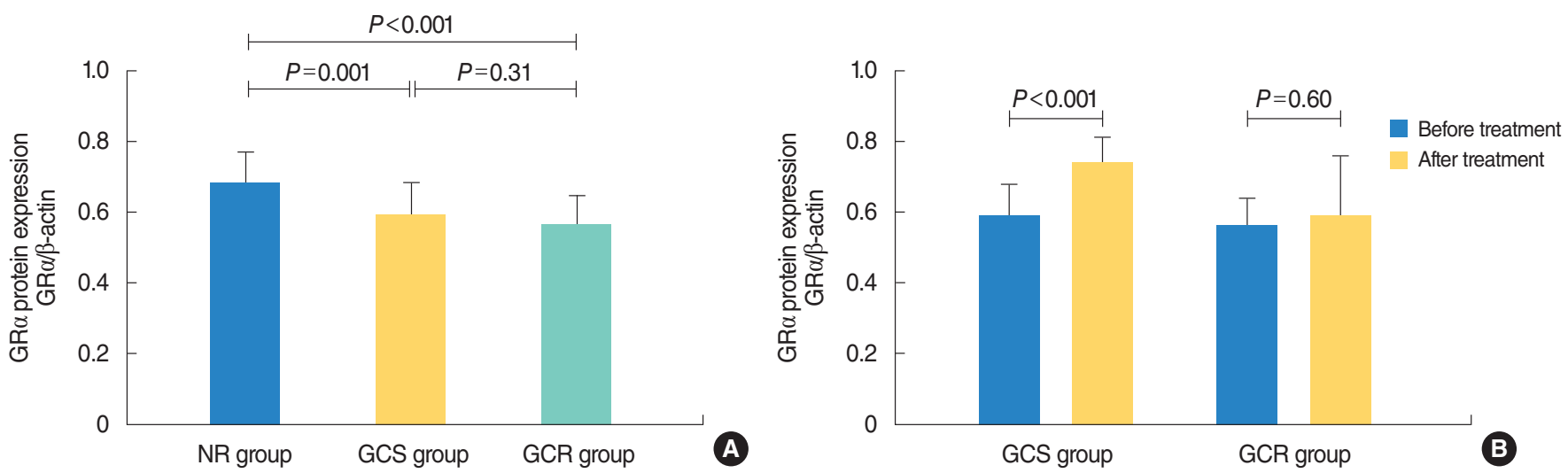

Fig. 4. (A) Glucocorticoid receptor $\alpha(\mathrm{GR} \alpha)$ protein in the normal reference (NR) group and sudden sensorineural hearing loss (SSNHL) patients before glucocorticoid (GC) treatment. Prior to treatment, significantly lower GRa protein levels were observed in the glucocorticoid-sensitive (GCS) and glucocorticoid-resistant (GCR) groups compared to the NR group $(P=0.001$ or $P<0.05)$, with the lowest GR $\alpha$ protein levels observed in the GCR group. (B) Comparison of GR $\alpha$ protein level in the GCS and GCR groups before and after GC treatment. After GC treatment, GR $\alpha$ protein levels significantly increased in the GCS group $(P<0.001)$, while no significant change was observed in the GCR group $(P=$ 0.60). (A) Comparison between groups using one-way analysis of variance test. (B) Comparison before and after treatment using paired-sample test.

\section{Increased protein levels of GR $\alpha$ and HDAC2 after GC treatment}

The relative protein levels of GR $\alpha$, GR $\beta$, and HDAC2 were calculated and normalized against the internal $\beta$-actin standard among 20 NR, 37 GCS, and 18 GCR subjects. Before GC treatment, significantly lower GR $\alpha$ protein levels were observed in the GCR and the GCS groups compared to the NR group $(0.56 \pm$ 0.09 in the GCR group, $0.59 \pm 0.10$ in the GCR group, $0.68 \pm$ 0.09 in the NR group) (Figs. 3, 4A). The levels of GR $\alpha$ were significantly increased in the GCS group after GC treatment $(P<$ $0.001)$, while no change was observed in the GCR group $(P=$ 0.60 ) (Figs. 3, 4B). No GR $\beta$ protein was detected in any sample we tested.

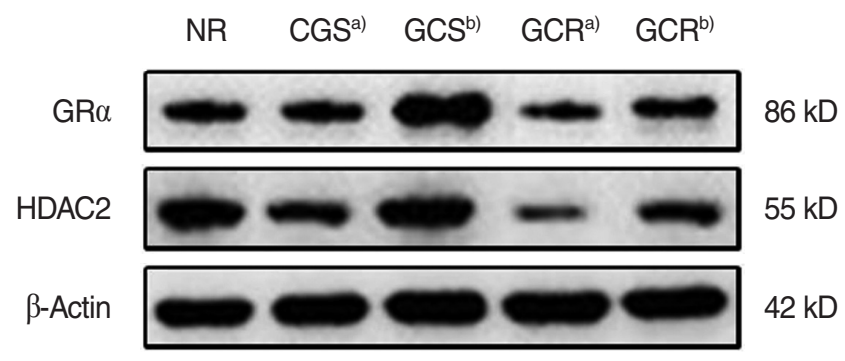

Fig. 3. Western blotting for glucocorticoid receptor $\alpha(\mathrm{GR} \alpha)(86 \mathrm{kD})$ and histone deacetylase 2 (HDAC2; $55 \mathrm{kD}$ ) in the normal reference (NR), glucocorticoid-sensitive (GCS) and glucocorticoid-resistant (GCR) groups. Each lane represents one sample from each group.

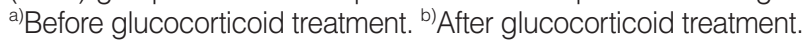



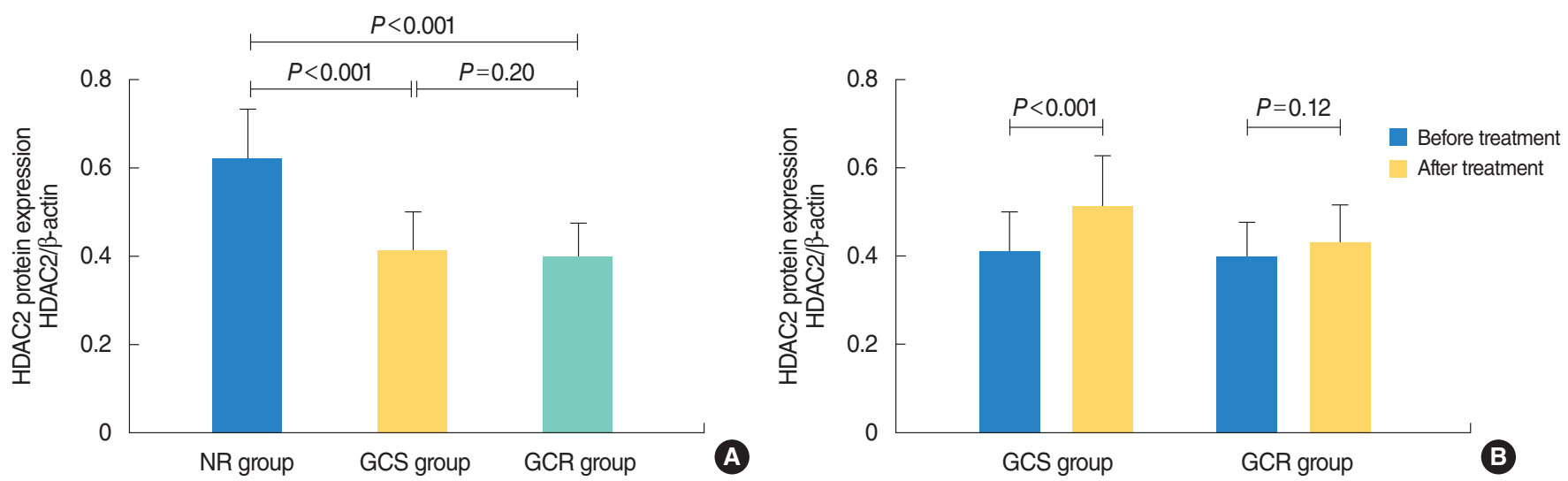

Fig. 5. (A) Histone deacetylase 2 (HDAC2) protein in the normal reference (NR) group and sudden sensorineural hearing loss (SSNHL) patients before glucocorticoid (GC) treatment. Prior to GC treatment, HDAC2 protein levels were significantly lower in SSNHL patients compared to the NR group $(P<0.001)$. (B) Comparison of HDAC2 protein levels in the glucocorticoid-sensitive (GCS) and glucocorticoid-resistant (GCR) groups before and after GC treatment. After glucocorticoid $(\mathrm{GC})$ treatment, HDAC2 protein levels significantly increased in the GCS group $(P<0.001)$, while no significant change was observed in the GCR group $(P=0.12)$. (A) Comparison between groups using one-way analysis of variance test. (B) Comparison before and after treatment using paired-sample test.
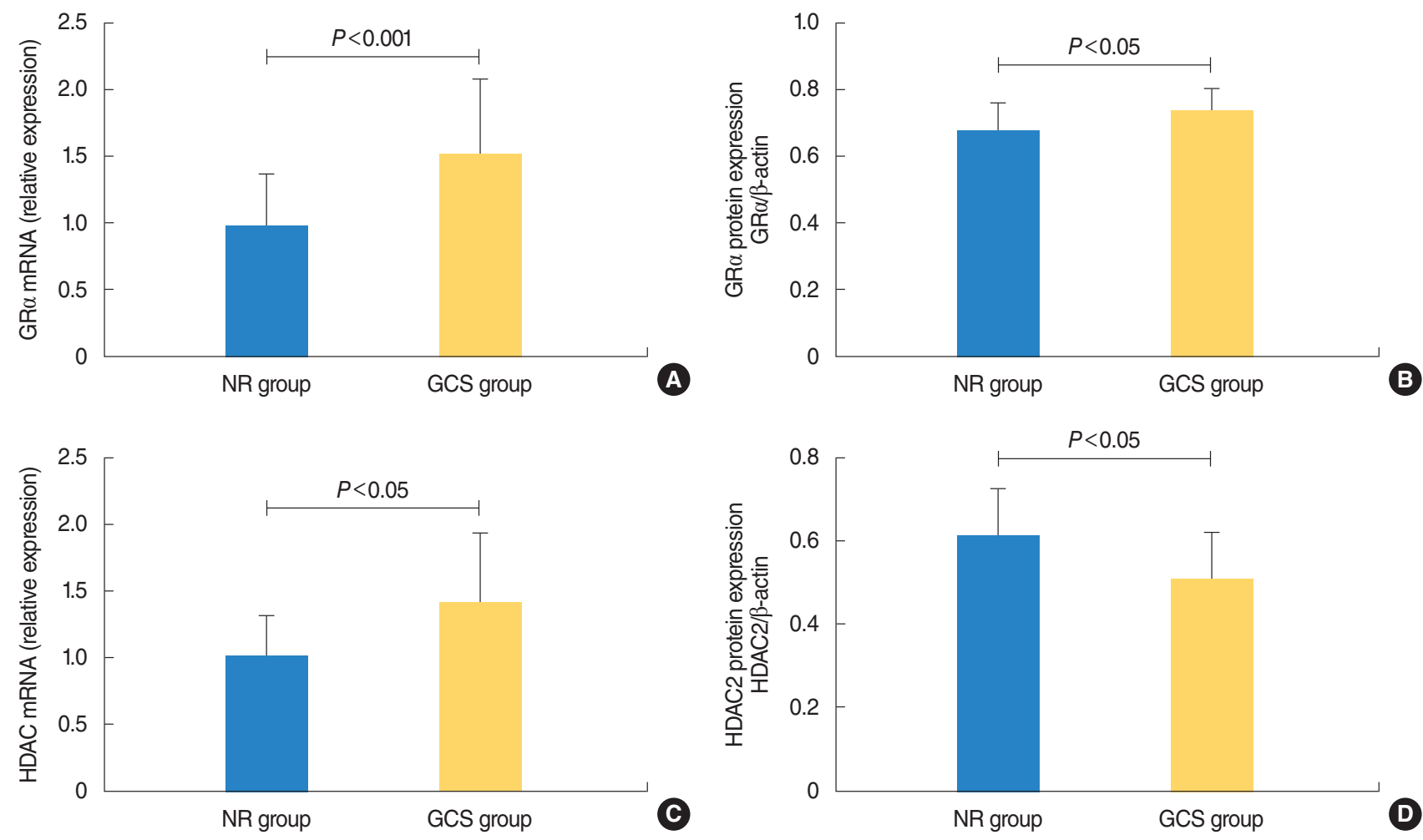

Fig. 6. The comparison of the expression levels of glucocorticoid receptor $\alpha(G R \alpha)(A, B)$ and histone deacetylase 2 (HDAC2) (C, D) between the normal reference (NR) group and the glucocorticoid-sensitive (GCS) group after treatment. After glucocorticoid (GC) treatment, the mRNA level of GR $\alpha$ and HDAC2 in GCS group significantly increased and were higher compared with NR group $(P<0.001$ or $P=0.05)$. The protein level of GRa in GCS group also significantly increased $(P<0.05)$ and were higher compared with NR group. The protein level of HDAC2 also increased after treatment but were still lower compared with NR group $(P<0.05)$. Comparison between groups using independent samples t-test.

As shown in Fig. 5A, HDAC2 protein level was $0.41 \pm 0.09$ in the GCS group, $0.40 \pm 0.08$ in the GCR group, much lower than $0.62 \pm 0.12$ in the NR group before GC treatment. After GC treatment, HDAC2 protein level was significantly increased in the GCS group $(P<0.001)$ (Figs. 3, 5B), while no change was observed in the GCR group $(P=0.12)$ (Figs. 3, 5B). 


\section{Comparison of expression levels of GR $\alpha$ and HDAC2 between NR and GCS group after treatment}

After GC treatment, the expression levels of GR $\alpha$ and the mRNA level of HDAC2 in GCS group significantly increased and were higher compared with NR group $(P<0.001$ or $P=0.05)$ (Fig. 6A$C)$. The protein level of HDAC2 also increased after treatment but were still lower compared with NR group $(P<0.05)$ (Figs. 5B, $6 \mathrm{D})$. So the ability of increased expressions of GR and HADC2 after GC treatment may be the critical role for GC sensitivity in SSNHL, irrespective to their expression levels compared to NR.

\section{DISCUSSION}

The molecular mechanisms underlying steroid resistance remain unclear. However, it has been proposed that reduced expression and/or activity of GR $\alpha$ and HDAC2 may play an important role in GC insensitivity $[10,13,19]$. It has been suggesting that reduced HDAC2 may be caused by increased expression of GR $\beta$ in asthma and chronic obstructive pulmonary diseases although conflicting reports are found in literature $[11,12,19]$. Our previous studies have suggested that reduced HDAC2 is also associated with steroid resistance in refractory SSNHL patients [15]. In the present study, we have extended these evaluations by examining GR $\alpha$, GR $\beta$, and HDAC2 gene expression and protein levels in PBMCs of patients with severe or profound SSNHL before and after GC treatment. We demonstrated that both the mRNA and protein levels of GR $\alpha$ and HDAC2 were significantly increased in patients who had significant hearing improvement after GC therapy, while no changes were observed in GCR patients. These data suggest that GR $\alpha$ and HDAC2 may be critical factors for mediating, or at the very least predicting, GC sensitivity in SSNHL patients.

In the present study, the GR $\alpha$ and HDAC2 mRNA level before GC treatment was identical in the GC-resistant, GC-sensitive and NR groups while HDAC2 protein levels were significantly lower in all SSNHL patients than the NR group. The discrepancies between mRNA and protein expression levels could be explained by posttranslational regulation [15]. Some factors in the pathogenesis of SSNHL, such as oxidative stress or inflammation, could affect the posttranslational regulation of GR and HDAC2 [20-22]. Lower protein levels of GR and HDAC2 expression might be evoked by a destabilization of mRNA and/or by the induction of ubiquitination and proteasomal degradation [20]. Since both GCS and GCR groups had reduced protein levels of GR $\alpha$ and HDAC2 before treatments, the reduced GR $\alpha$ and HDAC2 could be causative factors of SSNHL as well as the prognostic factors of SSNHL. However, we believe that the reduced GR $\alpha$ and HDAC2 are closely related to GC insensitivity in SSNHL patients [15] as shown in other systems [23]. The potential molecules causing reduction of HDAC2 and GR $\alpha$ in SSNHL may include nitric oxide (NO), free radicals, as well as phosphoinositide-3-kinase (PI3K) $\delta$ and erythroid-derived-2 like 2 (Nrf2) that can be activated by oxidative stress [24].

However, immediately after GC therapy, significant elevations in HDAC2 mRNA and protein levels were uniquely observed in the GCS group. These data suggest that no or low response of HDAC2 to steroid treatment in GC-resistant SSNHL patients may play a fundamental mechanistic role in GC insensitivity, which is consistent with our previous report [15]. HDAC2 is a critical component of the GC-GR complex that mediates the transrepression of NF-KB transcriptional activity by deacetylating histones in the proinflammatory genes and by deacetylating GR [19]. In steroid-resistant asthma patients, HDAC2 expression is greatly reduced in alveolar macrophages and PBMCs [25].The efficacy of GCs was affected when HDAC2 were knockdown [21]. Both PI3K $\delta$ inhibitors and Nrf2 activators can increase HDAC2 expression and reverse steroid resistant [24]. The potential molecular mechanism of HDAC2 level or activity reduction in SSNHL is that oxidative stress activates phosphoinositide3-kinase $\delta$, and the latter leads to phosphorylation and inactivation of HDAC2. On the other hand, the reduction of Nrf2 is also linked to reduced HDAC2 expression by preventing HDAC2 deacetylation and activation [24]. Therefore, in light of our current results, the manner in which these factors contextually impact GC resistance in SSNHL patients, bears further evaluation.

Significant elevations in GR $\alpha$ mRNA and protein levels were also observed in the GCS group immediately after GC therapy. These data suggest that, besides HDAC2, low level of GR $\alpha$ in GC-resistant SSNHL patients may also play a fundamental mechanistic role in GC insensitivity, GRa is thought to be the main mediator of GC action [7]. A high GRa level is essential for GC response as demonstrated in various cell lines [26].

The mRNA and protein levels of GR $\beta$ were very low or undetectable in PBMCs in all SSNHL patients. Although the GR $\beta$ mRNA expression in PBMCs was slightly increased in all patients after GC treatment, no significant difference was observed. These results suggest that GR $\beta$ may not be playing a significant role in steroid resistance in SSNHL. Consistent with our results, Hausmann et al. [27] and Butler et al. [12] did not find elevated GR $\beta$ mRNA level in PBMCs or lung epithelial cells in steroid-resistant asthma patients. High levels of GR $\alpha$ with very low levels of GR $\beta$ were also observed in adult immune thrombocytopenia, suggesting GR $\beta$ may not be involved in steroid resistance [13]. Given the substantially higher expression of GR $\alpha$, GR $\beta$ is unlikely to function as a dominant-negative isoform of GR $\alpha$ and likely has little to do with the steroid resistance [12]. Therefore, targeted modulation of GR $\alpha$ levels could be used as a therapeutic strategy to overcome GC resistance [21].

Our results have demonstrated that the relative response of $\mathrm{GR} \alpha$ and HDAC2 to steroid treatment may predict the prognosis of hearing improvement in SSNHL patients. Our data have also indicated that GR $\beta$ may not play a significant role in steroid resistance in patients with severe SSNHL. 


\section{CONFLICT OF INTEREST}

No potential conflict of interest relevant to this article was reported.

\section{ACKNOWLEDGMENTS}

This study was supported by a grant from the National Natural Science Funds of China (81271074); a grant for clinical medicine from the science and technology department of Jiangsu Province, China (BL2014002) and a grant (WSN-009) from the Six Talent Peaks Project of Jiangsu Province, China, and Jiangsu Provincial Key Medical Discipline (ZDXKB2016015), China.

The authors wish to thank Drs. Xiaoping Du and Matthew B. West (Hough Ear Institute, Oklahoma, USA) for critically reviewing the manuscript.

\section{ORCID}

Xiuling Zhang https://orcid.org/0000-0002-7902-5870

Wandong She https://orcid.org/0000-0003-1923-8687

\section{AUTHOR CONTRIBUTIONS}

Conceptualization: WS. Data curation: XZ, ZG. Formal analysis: XZ,WS, JC. Funding acquisition:WS, JC. Methodology: XZ, ZG, HQ. Project administration: XZ, JC, HQ, YD. Visualization: XZ, YD. Writing - original draft: XZ.Writing - review \& editing:WS, $\mathrm{XZ}, \mathrm{YD}$.

\section{REFERENCES}

1. El Sabbagh NG, Sewitch MJ, Bezdjian A, Daniel SJ. Intratympanic dexamethasone in sudden sensorineural hearing loss: a systematic review and meta-analysis. Laryngoscope. 2017 Aug;127(8):1897-908.

2. Trune DR, Canlon B. Corticosteroid therapy for hearing and balance disorders. Anat Rec (Hoboken). 2012 Nov;295(11):1928-43.

3. Stachler RJ, Chandrasekhar SS, Archer SM, Rosenfeld RM, Schwartz SR, Barrs DM, et al. Clinical practice guideline: sudden hearing loss. Otolaryngol Head Neck Surg. 2012 Mar;146(3 Suppl):S1-35.

4. Michel O; Deutsche Gesellschaft fur Hals-Nasen-Ohren-Heilkunde, Kopf- und Hals-Chirurgie. The revised version of the German guidelines "sudden idiopathic sensorineural hearing loss". Laryngorhinootologie. 2011 May;90(5):290-3.

5. Gul F, MuderrisT, Yalciner G, Sevil E, Bercin S, Ergin M, et al. A comprehensive study of oxidative stress in sudden hearing loss. Eur Arch Otorhinolaryngol. 2017 Mar;274(3):1301-8.

6. Niu N, Manickam V, Kalari KR, Moon I, Pelleymounter LL, Eckloff BW, et al. Human glucocorticoid receptor alpha gene (NR3C1) pharmacogenomics: gene resequencing and functional genomics. J Clin Endocrinol Metab. 2009 Aug;94(8):3072-84.
7. Pujols L, Mullol J, Roca-Ferrer J,Torrego A, Xaubet A, Cidlowski JA, et al. Expression of glucocorticoid receptor alpha- and beta-isoforms in human cells and tissues. Am J Physiol Cell Physiol. 2002 Oct; 283(4):C1324-31.

8. Rarey KE, Curtis LM. Receptors for glucocorticoids in the human inner ear. Otolaryngol Head Neck Surg. 1996 Jul;115(1):38-41.

9. She W, Dai Y, Du X, Yu C, Chen F, Wang J, et al. Hearing evaluation of intratympanic methylprednisolone perfusion for refractory sudden sensorineural hearing loss. Otolaryngol Head Neck Surg. 2010 Feb;142(2):266-71.

10. Yang N, Ray DW, Matthews LC. Current concepts in glucocorticoid resistance. Steroids. 2012 Sep;77(11):1041-9.

11. Li LB, Leung DY, Martin RJ, Goleva E. Inhibition of histone deacetylase 2 expression by elevated glucocorticoid receptor beta in steroidresistant asthma. Am J Respir Crit Care Med. 2010 Oct;182(7):87783

12. Butler CA, McQuaid S, Taggart CC, Weldon S, Carter R, Skibinski G, et al. Glucocorticoid receptor $\beta$ and histone deacetylase 1 and 2 expression in the airways of severe asthma. Thorax. 2012 May;67(5): 392-8.

13. Ma L, Fang M, Liang Y, Xiang Y, Jia Z, Sun X, et al. Low expression of glucocorticoid receptor alpha isoform in adult immune thrombocytopenia correlates with glucocorticoid resistance. Ann Hematol. 2013 Jul;92(7):953-60.

14. Ito K, Yamamura S, Essilfie-Quaye S, Cosio B, Ito M, Barnes PJ, et al. Histone deacetylase 2-mediated deacetylation of the glucocorticoid receptor enables NF-kappaB suppression. J Exp Med. 2006 Jan; 203(1):7-13.

15. Hou J, She W, Du X, Dai Y, Xie L, Zhou Q. Histone Deacetylase 2 in sudden sensorineural hearing loss patients in response to intratympanic methylprednisolone perfusion. Otolaryngol Head Neck Surg. 2016 Jan;154(1):164-70.

16. Editorial Board of Chinese Journal of Otorhinolaryngology Head and Neck Surgery; Society of Otorhinolaryngology Head and Neck Surgery, Chinese Medical Association. Guideline of diagnosis and treatment of sudden deafness (2015). Zhonghua Er Bi Yan Hou Tou Jing Wai Ke Za Zhi. 2015 Jun;50(6):443-7.

17. Livak KJ, Schmittgen TD. Analysis of relative gene expression data using real-time quantitative PCR and the 2(-Delta Delta C(T)) Method. Methods. 2001 Dec;25(4):402-8.

18. $\mathrm{Yu} \mathrm{H}, \mathrm{Li} \mathrm{H}$.Association of vertigo with hearing outcomes in patients with sudden sensorineural hearing loss: a systematic review and metaanalysis. JAMA Otolaryngol Head Neck Surg. 2018 Aug;144(8): 677-83.

19. Barnes PJ. Corticosteroid resistance in patients with asthma and chronic obstructive pulmonary disease. J Allergy Clin Immunol. 2013 Mar;131(3):636-45.

20. Heinrich UR, Strieth S, Schmidtmann I, Stauber R, Helling K. Dexamethasone prevents hearing loss by restoring glucocorticoid receptor expression in the guinea pig cochlea. Laryngoscope. 2016 Jan; 126(1):E29-34

21. Li J, Liu D, Wu J, Zhang D, Cheng B, Zhang Y, et al. Ginsenoside Rg1 attenuates ultraviolet B-induced glucocortisides resistance in keratinocytes via Nrf2/HDAC2 signalling. Sci Rep. 2016 Dec;6:39336.

22. Yang SR, Chida AS, Bauter MR, Shafiq N, Seweryniak K, Maggirwar $\mathrm{SB}$, et al. Cigarette smoke induces proinflammatory cytokine release by activation of NF-kappaB and posttranslational modifications of histone deacetylase in macrophages. Am J Physiol Lung Cell Mol Physiol. 2006 Jul;291(1):L46-57.

23. Wilkinson L, Verhoog NJD, Louw A. Disease and treatment associated acquired glucocorticoid resistance. Endocr Connect. 2018 Dec; 7(12):R328-49.

24. Mercado N, Thimmulappa R, Thomas CM, Fenwick PS, Chana KK, Donnelly LE, et al. Decreased histone deacetylase 2 impairs Nrf2 
activation by oxidative stress. Biochem Biophys Res Commun. 2011 Mar;406(2):292-8.

25. Hew M, Bhavsar P,Torrego A, Meah S, Khorasani N, Barnes PJ, et al. Relative corticosteroid insensitivity of peripheral blood mononuclear cells in severe asthma. Am J Respir Crit Care Med. 2006 Jul;174(2): 134-41.

26. Riml S, Schmidt S, Ausserlechner MJ, Geley S, Kofler R. Glucocorti- coid receptor heterozygosity combined with lack of receptor autoinduction causes glucocorticoid resistance in Jurkat acute lymphoblastic leukemia cells. Cell Death Differ. 2004 Jul;11 Suppl 1:S65-72. 27. Hausmann M, Herfarth H, Scholmerich J, Rogler G. Glucocorticoid receptor isoform expression does not predict steroid treatment response in IBD. Gut. 2007 Sep;56(9):1328-9. 